\title{
Nylon-6 based nanocomposite films for capacitor applications
}

\author{
Amoghavarsha Mahadevegowda*, Colin Johnston, and Patrick S Grant
}

\begin{abstract}
Nylon-6 and Al based nanocomposite films were fabricated via a scalable vacuum co-deposition technique. The relative deposition rates of the constituent phases - nylon-6 (matrix) and Al (filler) - were varied systematically to yield films of different compositions and their dielectric properties, particularly the measured dielectric constants $k$, were compared with predictions of effective medium expressions. The effect of absorbed water, temperature and heat treatment on $k$ of the nano-films were studied. $X$-ray photoelectron spectroscopy revealed the presence of an Al-based oxide, which was correlated to the observed enhancement in the dielectric properties of the nanocomposites. The effect of the relative deposition rates of the constituent phases on $k$ and the chemistry of the deposited films fabricated via co-deposition was studied and explained using X-ray photoelectron spectroscopy results.
\end{abstract}

\section{INTRODUCTION}

Polymer-based nanocomposites (PNCs) [1] consisting of a comparatively polarisable filler embedded in a polymer matrix are promising candidates for capacitor applications because they can be processed into large area thin-films with attractive dielectric properties such as high dielectric constant $k$ and breakdown strength [2], [3], [4] while maintaining low loss [5], [6]. However, difficulties involved in scaling-up PNC processing techniques such as spin coating and multistep chemical synthesis routes have limited the large-scale commercial manufacturing of high- $k$ PNCs.

Vacuum based deposition techniques, which have the potential to be scaled up, for example in a roll-to-roll coater [7], [8], provide an alternative route to large scale manufacturing of thin-film high- $k$ dielectrics. These vacuumbased processes can be adapted to produce composite films by co-deposition from two or more sources in which the volume fraction $v_{f}$ of the constituent phases is controlled by varying the relative deposition rates. If good control over the relative deposition rates can be achieved, co-deposition facilitates a three-dimensional and homogeneous distribution of the minority phase. Co-deposition, in principle, could also allow processing of a multi-material composite three or more materials by evaporating them simultaneously.

In this work, we explore co-deposition as a technique to fabricate polymer-nanocomposites with enhanced dielectric response over polymer only equivalents. Aluminium was chosen as the filler because $\mathrm{Al}$ is highly reactive and readily forms a relatively high- $k$ oxide [2], [9], [10] ( $k$ of bulk alumina $\sim 9$ ), and is widely used in metallisation via roll-toroll processing. Nylon- 6 was used as the matrix because of

Amoghavarsha Mahadevegowda, Colin Johnston, and Patrick Grant are with Department of Materials, University of Oxford, 16 Parks Road, Oxford OX1 3PH, United Kingdom.

*Email-id: amogh.gowda@materials.ox.ac.uk the comparative ease of its processability by evaporation and wide-spread applicability. Both $\mathrm{Al}$ and nylon- 6 are also relatively cheap materials. The variation of the dielectric properties with $v_{f}$ of the minority Al-rich phase was then studied and compared with the predictions of effective medium expressions. X-ray photoelectron spectroscopy (XPS) was carried out to investigate the presence of any aluminiumbased oxides and their role in enhancing the effective $k$ of the nanocomposite. The effect of the relative deposition rates of the parent phases on the chemistry of the deposited film was studied in light of our previously published work [2], [11] and explained using the XPS results. The influence of absorbed water, temperature and the extent of oxidation of $\mathrm{Al}$ was correlated with enhanced dielectric properties, and the merits of co-deposition as a fabrication technique are discussed.

\section{EXPERIMENTAL}

\section{A. Fabrication}

PNC films were fabricated by co-evaporation of aluminium and nylon-6 in an Edwards Auto 306 Cryo evaporator, where the evaporator-chamber was evacuated to a pressure of $5 \times 10^{-4} \mathrm{~Pa}$ before deposition.

An aluminium layer (thickness $t \sim 40 \mathrm{~nm}$ ) was deposited on a glass substrate by thermally evaporating Al wire $(99.999 \%$ pure, Alfa Aesar, $0.5 \mathrm{~mm}$ diameter) from a $\mathrm{W}$ boat at a deposition rate $\dot{t}=0.5 \mathrm{~nm} / \mathrm{s}$ where $\dot{t}_{A l}$ and $t$ were measured in real-time using a quartz-crystal thickness-monitor. The deposited Al layer provided the "bottom" electrode of a simple parallel-plate capacitor. Then $\mathrm{Al}$ and nylon6 (Aldrich) were co-evaporated and the deposition rate of nylon-6 was maintained at $\dot{t}_{N y} \sim 0.1 \mathrm{~nm} / \mathrm{s}$ while varying the $\mathrm{Al}$ deposition rate to obtain different volume fractions of $\mathrm{Al}$ (filler) in the nylon- 6 matrix. Then a top Al layer $(40 \mathrm{~nm}$ thick electrode) was deposited to complete the parallel plate capacitor arrangement.

\section{B. Characterisation}

X-ray photoelectron spectroscopy (XPS) was performed in a VG Microtech CLAM 4 MCD analyser system using 200 Watt unmonochromated Mg X-ray excitation to study the chemical composition of the filler. The dielectric properties were investigated using a Solartron SI 1260 impedance analyzer operating at $1 \mathrm{kHz}$. The PNC-based capacitors were dried at $40{ }^{\circ} \mathrm{C}$ for 20 hours to minimise the effect of absorbed water immediately before dielectric characterisation. The thickness of the PNC films was measured using a DekTak 6M profilometer (Veeco Instruments, Inc), and $k$ was estimated directly from the impedance data. 


\section{RESULTS AND DISCUSSION}

The dielectric response of the PNCs at $40{ }^{0} \mathrm{C}$ was studied and compared with some of the most popular analytical models from literature [12], [13], [14], [15]:

Rule of mixtures:

$$
\epsilon_{e f f}=v_{f_{1}} \epsilon_{1}+v_{f_{2}} \epsilon_{2}
$$

Lichtenecker logarithmic mixing law:

$$
\log \left(\epsilon_{e f f}\right)=v_{f_{1}}\left[\log \left(\epsilon_{1}\right)\right]+v_{f_{2}}\left[\log \left(\epsilon_{2}\right)\right]
$$

Maxwell's Equation:

$$
\epsilon_{e f f}=\epsilon_{1}\left(\frac{\epsilon_{1}+2 \epsilon_{1}-2\left(1-v_{f_{1}}\right)\left(\epsilon_{1}-\epsilon_{2}\right)}{\epsilon_{2}+2 \epsilon_{1}+\left(1-v_{f_{1}}\right)\left(\epsilon_{1}-\epsilon_{2}\right)}\right)
$$

where the subscripts 1 and 2 represent the two phases; $\epsilon$ and $v_{f}$ represent dielectric constant and volume fraction respectively. The assumed values for $\epsilon_{1}$ (alumina) [16], [17], [18] and $\epsilon_{2}$ (experimentally deduced dielectric constant of nylon- 6 at $40{ }^{0} \mathrm{C}$ ) were 9 and 4.3 respectively. All expressions in Equations 1 to 3 assume composite systems with uniformly dispersed, non-conducting particles.

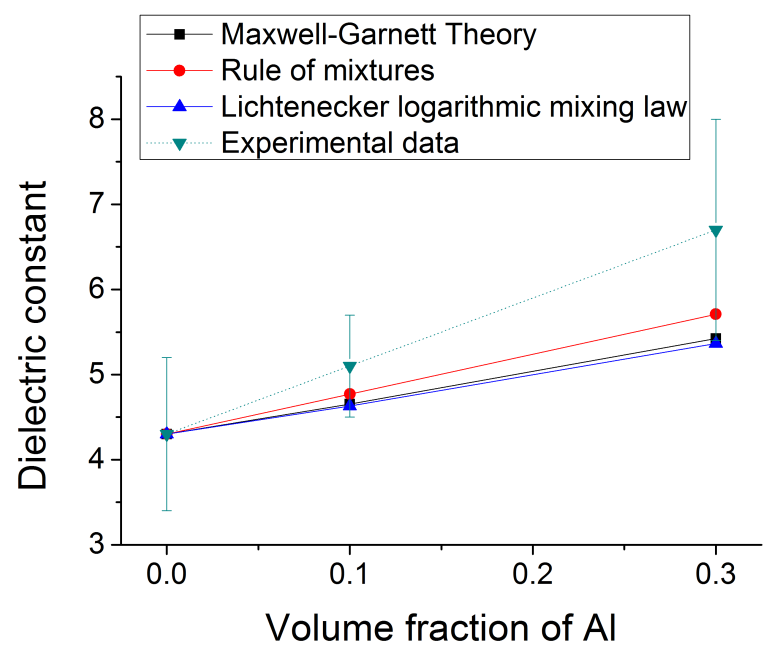

Fig. 1. A comparison between averaged experimental data at $40{ }^{0} \mathrm{C}$ and predictions of dielectric constant as a function of $v_{f}$ in PNCs at $1 \mathrm{kHz}$.

Figure 1 shows that the experimental data were consistently above the effective dielectric constants suggested by commonly used expressions, which was initially attributed to possible trapped water at the interface between the filler and the matrix in the PNCs even after drying for $20 \mathrm{~h}$ at 40 ${ }^{0} \mathrm{C}$.

Absorbed water can remain in small quantities even after prolonged drying for 100 hours because it is strongly bound to the polar sites [19], [20]. So although "pure" polymers are known to be low-loss dielectrics, the presence of such strongly bound water increases the dielectric loss in "impure" polymers.

Increasing temperature in the presence of water molecules may lead to an increase in the number of "polarisable entitites" such as relatively mobile and polarisable polymersegments [21], [22], [23], which result in an enhancement in $k$. The presence of defective sites in the polymer due to the condensation processing co-deposition and the incorporation of a filler (in this case, $\mathrm{Al}$ ) may have also helped produce a conducive environment for absorbing and retaining water in the PNC films, which again enhanced $k$.

Returning to Fig. 1, the plots of Equations 1 to 3 assumed that all the evaporated $\mathrm{Al}$ was in the form of alumina with a "bulk" dielectric constant. This assumed oxidation of $\mathrm{Al}$ might have taken place: (i) during the deposition process when $\mathrm{Al}$ reacted with $\mathrm{O}_{2}$ present in nylon- 6 and any residual chamber $\mathrm{O}_{2}$, or (ii) when the PNCs were taken out of the vacuum chamber and exposed to atmosphere since the thin (up to $200 \mathrm{~nm}$ ) nylon-6 films were unlikely to be entirely impermeable to atmospheric $\mathrm{O}_{2}$. The former case was investigated by calculating the amount of $\mathrm{O}_{2}$ in the chamber (see later), but no practical method for assessing the contribution of any reaction with nylon- 6 to oxidation was conceived.

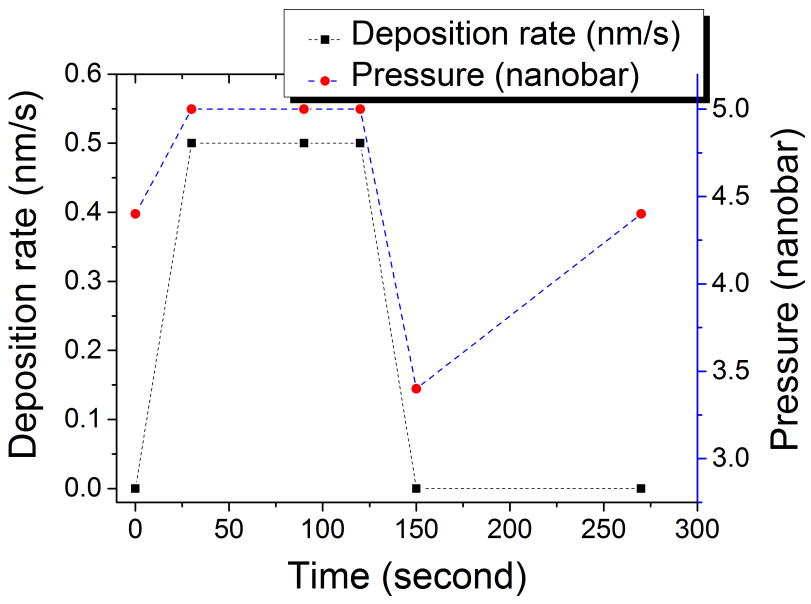

Fig. 2. The variation of deposition rate and chamber-pressure with time during Al evaporation. Lines connecting the data are only a guide to the eye.

Figure 2 shows a typical variation of deposition rate and chamber-pressure with time during $\mathrm{Al}$ evaporation. When evaporation of $\mathrm{Al}$ started, the chamber-pressure rose from 4.4 nanobar at $0 \mathrm{~s}$ and $0 \mathrm{~nm} / \mathrm{s}$ to 5.0 nanobar at $30 \mathrm{~s}$ and $0.5 \mathrm{~nm} / \mathrm{s}$, due to the presence of the Al-vapour. When Al-evaporation was stopped at $120 \mathrm{~s}$, the chamber-pressure very quickly reduced to 3.4 nanobar, which was lower than the initial pressure of the chamber, and which might be attributed to gettering of $\mathrm{O}_{2}$ due to the conversion of gaseous $\mathrm{Al}$ (vapour) to solid alumina or other oxides at deposition/condensation. The chamber-pressure then recovered to 4.4 nanobar within $120 \mathrm{~s}$ due to the unavoidable, continuous ingress of small amount of atmospheric air into the chamber (which presumably occurred throughout deposition). The net change in the chamber-pressure at $120 \mathrm{~s}$ is given by $[(4.4-3.4) / 4.4]^{*} 100=22.7 \%$, which is reasonable given the concentration of atmospheric $\mathrm{O}_{2}(21 \%)$. 
In order to estimate if the amount of oxygen present in the chamber was sufficient to oxidise all of the evaporated $\mathrm{Al}$, the ideal gas law was used:

$$
P V=n R T
$$

where $P$ is the absolute pressure of the gas $=10^{-4} \mathrm{~Pa}$, $V$ is the volume of the gas (in this case, the volume was approximated to be the volume of the chamber) $=0.1 \mathrm{~m}^{3}, n$ is the number of moles of the gas, $T$ is the absolute temperature of the gas ( $T$ was measured in-situ using a thermocouple $)=$ $293 \mathrm{~K}$, and $\mathrm{R}=8.314 \mathrm{~J} / \mathrm{mol} / \mathrm{K}$ is the ideal, or universal, gas constant. The number of moles of $\mathrm{O}_{2}$ present in the chamber was estimated at $\sim 1$ nanomole, which is $\sim 10^{6}$ less than the amount of $\mathrm{O}_{2}(\sim 1$ millimole $)$ required to oxidise the $\mathrm{Al}$ in the W-boat, $75 \mathrm{mg}$ (even if two-thirds of $75 \mathrm{mg}$ is assumed to be evaporated), to form stoichiometric $\mathrm{Al}_{2} \mathrm{O}_{3}$. It was concluded therefore that the majority of $\mathrm{Al}$ oxidation took place by reacting with $\mathrm{O}_{2}$ when the chamber was vented back to atmosphere.
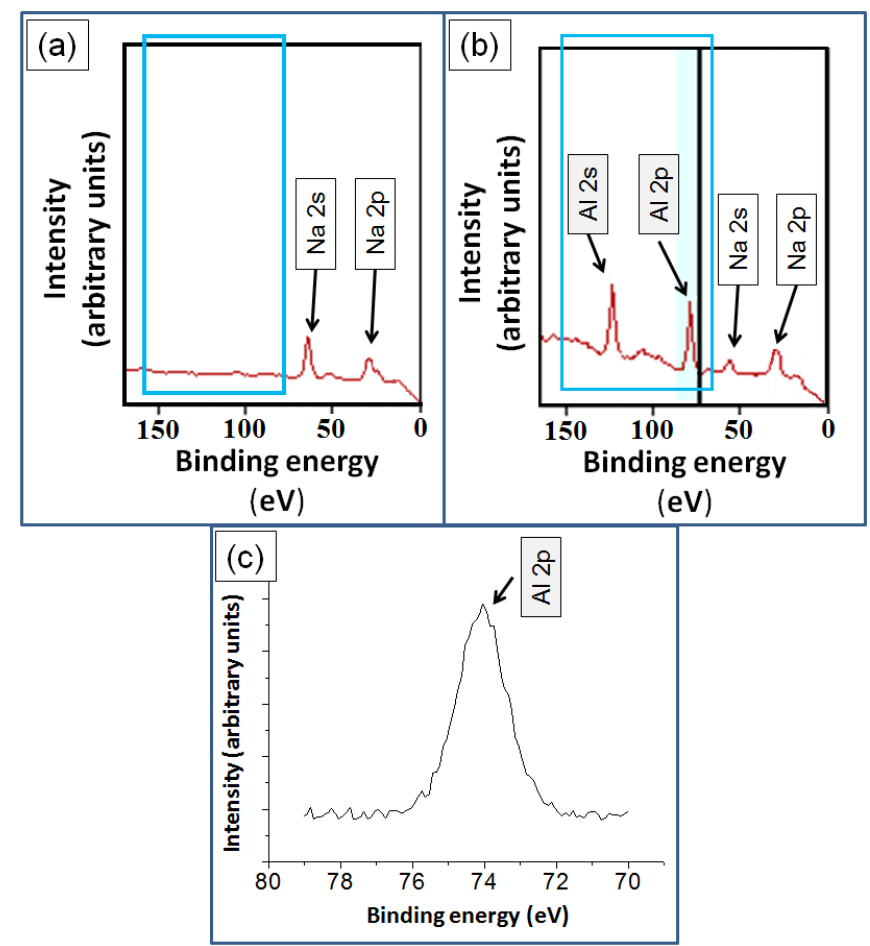

Fig. 3. X-ray photoelectron spectra of (a) a "pure" nylon-6 film, (b) a $\mathrm{PNC}_{30 \%}$ film, and (c) $\mathrm{PNC}_{30 \%}$ film showing detailed scan of the $\mathrm{Al} 2 \mathrm{p}$ peak. The $2 \mathrm{p}$ peak in (c) was at $74.04 \mathrm{eV}$, which is characteristic of $\mathrm{Al}_{2} \mathrm{O}_{3}$.

Experimental findings suggested the oxidation of $\mathrm{Al}$ and so qualitative post-deposition analysis was pursued in order to confirm the presence of $\mathrm{O}_{2}$ containing phases such as alumina $\left(\mathrm{Al}_{2} \mathrm{O}_{3}\right)$.

$\mathrm{X}$-ray photoelectron spectra from a "pure" nylon-6 film and a $\mathrm{PNC}_{30 \%}$ (i.e. 30 vol\% deposited $\mathrm{Al}$ ) film are shown in Figs. 3(a) and (b) respectively. Two $\mathrm{Al}$ peaks, $2 \mathrm{~s}$ and 2p, appeared only in Fig. 3(b) confirming the presence of Al in the nanocomposite film. In the spectrum from a detailed scan on the same PNC, shown in Fig. 3(c), the 2p peak appeared at $74 \mathrm{eV}$ which is characteristic of $\mathrm{Al}_{2} \mathrm{O}_{3}$ [24]. The characteristic peak of $\mathrm{Al}$ alone [24] at $71.8 \mathrm{eV}$ was not observed, again suggesting the complete oxidation of $\mathrm{Al}$, which is in contrast with our previous studies on nylon-6 based PNCs that were found to have Al-based nanoparticles with a metallic Al core and an Al-oxide shell, but which were fabricated via alternate deposition [2], [11].

In the co-deposited PNCs, Al is the minority phase with $\dot{t}_{A l}<0.1 \mathrm{~nm} / \mathrm{s}$ and $\dot{t}_{N y} \sim 0.1 \mathrm{~nm} / \mathrm{s}$ and led to the formation of relatively small clusters of $\mathrm{Al}$ atoms that oxidised completely unlike the $>12 \mathrm{~nm} \mathrm{Al}$ nanoparticles in our previous work [2] that only partially oxidised at the surface, resulting in self-passivation. Moreover the simultaneous or co-deposition of $\mathrm{Al}$ and nylon- 6 in this current work might have also prevented the formation of relatively large Al-based particles due to the continuous intermixing and overlapping of $\mathrm{Al}$ and nylon-6. So the Al distribution probably comprised a threedimensional embedding of oxidised Al-based "clusters" in the nylon-6 film during the co-deposition process, which resulted in the formation of PNCs with $k<9$ that is the upper limit in $k$ predicted by the effective medium expressions in Equations (1-3).

When compared with other material systems such as nearpercolating composites [25], [26], [27], [28] that exhibit $k \sim 10^{7}$ near the critical filler volume fraction, controlling the dielectric properties of our composites is relatively easy as they fall within the regime of effective medium expressions and $k$ is comparatively less sensitive to changes in the filler volume fraction. Percolating PNCs containing conductive fillers such as $\mathrm{Au}$ or $\mathrm{Ag}$ suffer from high dielectric loss and also are more difficult to process, especially on an industrial-scale, because it is hard to maintain the critical near-percolating volume fraction.

\section{CONCLUSIONS}

$\mathrm{Al}$ and nylon- 6 based nanocomposite films with elevated dielectric constant were successfully fabricated by a codeposition technique, and their dielectric properties were studied in comparison with analytical models. The relatively high $k$ in experimental data was ascribed to the presence of $\mathrm{Al}_{2} \mathrm{O}_{3}$, which was confirmed by XPS, and a combination of relatively high temperature and absorbed water molecules in the nanometer-thick PNC films. A drop in the chamberpressure during Al-evaporation was suggested to correspond with evaporated $\mathrm{Al}$ reacting with oxygen in-situ to form an Al-based oxide (supported by XPS spectra). However, a simple estimation suggested that the amount of oxygen present in the chamber was insufficient to oxidise all of the evaporated $\mathrm{Al}$ and suggested that remaining $\mathrm{Al}$ reacted with oxygen present in the nylon- 6 and most likely when the vacuum chamber was vented.

\section{REFERENCES}

[1] A. C. Balazs, T. Emrick, and T. P. Russell, "Nanoparticle polymer composites: Where two small worlds meet," Science, vol. 314, no. 5802, p. 1107, 2006.

[2] A. Mahadevegowda, N. P. Young, and P. S. Grant, "Coreshell nanoparticles and enhanced polarization in polymer based nanocomposite dielectrics," Nanotechnology, vol. 25, no. 47, p. 475706, 2014. 
[3] G. G. Raju, Dielectrics in Electrical Fields. Marcel Dekker, New York, 2003.

[4] A. Mahadevegowda, N. P. Young, and P. S. Grant, "Engineering the nanostructure of a polymer-nanocomposite film containing Ti-based core-shell particles to enhance dielectric response," Nanoscale, vol. 7, p. 15727, 2015.

[5] S. H. Lim, A. C. Rastogi, and S. B. Desu, "Electrical properties of metal-ferroelectric-insulator-semiconductor structures based on ferroelectric polyvinylidene fluoride copolymer film gate for nonvolatile random access memory application," Journal of Applied Physics, vol. 96 , no. 10, p. 5673, 2004.

[6] J. Xu, S. Bhattacharya, P. Pramanik, and C. P. Wong, "High dielectric constant polymer-ceramic (epoxy varnish-barium titanate) nanocomposites at moderate filler loadings for embedded capacitors," Journal of Electronic Materials, vol. 35, no. 11, p. 2009, 2006.

[7] Z. Ding, G. A. Abbas, H. E. Assender, J. J. Morrison, V. SanchezRomaguera, S. G. Yeates, and D. M. Taylor, "Improving the performance of organic thin film transistors formed on a vacuum flashevaporated acrylate insulator," Applied Physics Letters, vol. 103, no. $23,2013$.

[8] J. Affinito, P. Martin, M. Gross, C. Coronado, and E. Greenwell, "Vacuum deposited polymer metal multilayer films for optical application," Thin Solid Films, vol. 270, no. 1-2, p. 43, 1995.

[9] J. W. Xu, K. S. Moon, C. Tison, and C. P. Wong, "A novel aluminumfilled composite dielectric for embedded passive applications," IEEE Transactions on Advanced Packaging, vol. 29, no. 2, p. 295, 2006.

[10] J. Xu and C. P. Wong, "Characterization and properties of an organicinorganic dielectric nanocomposite for embedded decoupling capacitor applications," Composites Part A-Applied Science and Manufacturing, vol. 38, no. 1, p. 13, 2007.

[11] A. Mahadevegowda, N. P. Young, and P. S. Grant, "Electron microscopy of multi-layered polymer-nanocomposite based dielectrics," Journal of Physics: Conference Series, vol. 522, p. 012041, 2014.

[12] Y. G. Wu, X. H. Zhao, F. Li, and Z. G. Fan, "Evaluation of mixing rules for dielectric constants of composite dielectrics by MC-FEM calculation on 3D cubic lattice," Journal of Electroceramics, vol. 11, no. 3 , p. $227,2003$.

[13] X. Zhao, "Spray Deposition of Nanostructured Materials for Energy Storage," Ph.D. dissertation, University of Oxford, Oxford, UK, 2009.

[14] T. Yamada, T. Ueda, and T. Kitayama, "Piezoelectricity of a highcontent lead zirconate titanate polymer composite," Journal of Applied Physics, vol. 53, no. 6, p. 4328, 1982.

[15] D. H. Yoon, J. P. Zhang, and B. I. Lee, "Dielectric constant and mixing model of BaTiO3 composite thick films," Materials Research Bulletin, vol. 38 , no. 5 , p. $765,2003$.

[16] J. V. Florio, "Dielectric properties of alumina at high temperatures," Journal of the American Ceramic Society, vol. 43, no. 5, p. 262, 1960

[17] P. Katiyar, C. Jin, and R. J. Narayan, "Electrical properties of amorphous aluminum oxide thin films," Acta Materialia, vol. 53, no. 9, p. 2617, 2005.

[18] B. G. Segda, M. Jacquet, and J. P. Besse, "Elaboration, characterization and dielectric properties study of amorphous alumina thin films deposited by r.f. magnetron sputtering," Vacuum, vol. 62, no. 1, p. 27, 2001.

[19] L. L. Marsh, R. Lasky, D. P. Seraphim, and G. S. Springer, "Moisture solubility and diffusion in epoxy and epoxy-glass composites," IBM Journal of Research and Development, vol. 28, no. 6, p. 655, 1984.

[20] K. I. Ivanova, R. A. Pethrick, and S. Affrossman, "Investigation of hydrothermal ageing of a filled rubber toughened epoxy resin using dynamic mechanical thermal analysis and dielectric spectroscopy," Polymer, vol. 41, no. 18, p. 6787, 2000.

[21] J. D. Reid, W. H. Lawrence, and R. P. Buck, "Dielectric-properties of an epoxy-resin and its composite .1. Moisture effects on dipole relaxation," Journal of Applied Polymer Science, vol. 31, no. 6, p. 1771, 1986.

[22] L. L. Marsh, D. C. Vanhart, and S. M. Kotkiewicz, "A dielectric loss investigation of moisture in epoxy-glass composites," IBM Journal of Research and Development, vol. 29, no. 1, p. 18, 1985.

[23] P. Gonon, A. Sylvestre, J. Teysseyre, and C. Prior, "Combined effects of humidity and thermal stress on the dielectric properties of epoxysilica composites," Materials Science and Engineering B-Solid State Materials for Advanced Technology, vol. 83, no. 1-3, p. 158, 2001.

[24] B. R. Strohmeier, "An ESCA method for determining the oxide thickness on aluminum-alloys," Surface And Interface Analysis, vol. 15, no. 1, p. 51, 1990.
[25] H. Takele, S. Jebril, T. Strunskus, V. Zaporojchenko, R. Adelung, and F. Faupel, "Tuning of electrical and structural properties of metalpolymer nanocomposite films prepared by co-evaporation technique," Applied Physics A, vol. 92, no. 2, p. 345, 2008.

[26] F. He, S. Lau, H. L. Chan, and J. Fan, "High dielectric permittivity and low percolation threshold in nanocomposites based on poly(vinylidene fluoride) and exfoliated graphite nanoplates," Advanced Materials, vol. 21 , no. 6 , p. 710,2009

[27] X. Zhao, A. A. Koos, B. T. T. Chu, C. Johnston, N. Grobert, and P. S. Grant, "Spray deposited fluoropolymer/multi-walled carbon nanotube composite films with high dielectric permittivity at low percolation threshold," Carbon, vol. 47, no. 3, p. 561, 2009.

[28] Z. M. Dang, Y. H. Lin, and C. W. Nan, "Novel ferroelectric polymer composites with high dielectric constants," Advanced Materials, vol. 15 , no. 19 , p. $1625,2003$. 